\title{
Better than Expected Prognosis of Minimal Tracheal Invasion in Well Differentiated thyroid carcinoma
}

Narin Nard-Carmel ${ }^{1}$, Dana Amsterdam ${ }^{1}$, Anton Warshavsky ${ }^{1}$, Maya Ish Shalom ${ }^{1}$, Avraham Abergel $^{1}$, Anat Wengier ${ }^{2}$, Barak Ringel ${ }^{2}$, Elena Izhakov ${ }^{1}$, Dan Fliss ${ }^{2}$, and Gilad Horowitz ${ }^{2}$

${ }^{1}$ Tel Aviv Ichilov-Sourasky Medical Center

${ }^{2}$ Tel Aviv Sourasky Medical Center

April 28, 2020

\begin{abstract}
Background: The aim of this study was to compare outcomes of patients with minimal tracheal invasion undergoing shave resection to other forms of ETE in lieu of the new American Joint Committee on Cancer (AJCC) staging. Methods: All patients diagnosed with well differentiated thyroid cancer (WDTC) and ETE were analyzed. Structural recurrence was measured and compared between various forms of ETE. Results: A total of 132 patients were included in the study. Twenty-seven patients (20.4\%) had strap muscle invasion, twenty-two patients (16.7\%) had minimal tracheal invasion and 83 patients (62.9\%) had other forms of ETE. Kaplan-Meier models for structural recurrence revealed that when combining T3b patients with T4a shave patients and comparing them to other T4a patients, the former performed better than the latter $(\mathrm{p}=0.028 \mathrm{for}$ all ages; $\mathrm{p}=$ 0.021; for patients $>55$ years). Conclusions: Patients with minimal tracheal may be more correctly staged as T3b rather than T4a.
\end{abstract}

Better than Expected Prognosis of Minimal Tracheal Invasion in Well Differentiated thyroid carcinoma

\section{ABSTRACT}

Background:

The aim of this study was to compare outcomes of patients with minimal tracheal invasion undergoing shave resection to other forms of ETE in lieu of the new American Joint Committee on Cancer (AJCC) staging.

Methods:

All patients diagnosed with well differentiated thyroid cancer (WDTC) and ETE were analyzed. Structural recurrence was measured and compared between various forms of ETE.

Results:

A total of 132 patients were included in the study. Twenty-seven patients (20.4\%) had strap muscle invasion, twenty-two patients $(16.7 \%)$ had minimal tracheal invasion and 83 patients $(62.9 \%)$ had other forms of ETE. Kaplan-Meier models for structural recurrence revealed that when combining T3b patients with T4a shave patients and comparing them to other T4a patients, the former performed better than the latter $(\mathrm{p}=0.028$ for all ages; $\mathrm{p}=0.021$; for patients $>55$ years).

Conclusions:

Patients with minimal tracheal may be more correctly staged as T3b rather than T4a. 


\section{Introduction}

The majority of patients with thyroid carcinoma have well-differentiated disease limited to the thyroid gland. However, $6-13 \%$ of these tumors can present with extra-thyroid extension (ETE). [1, 2] ETE has been wellrecognized as a poor prognostic indicator, with 10-year overall survival (OS) rates dropping by more than $50 \%$ compared to tumors without ETE [3].

Tracheal invasion is a specific form of ETE and it is often recognized only at the time of surgery. [4] Surgical techniques for treating tracheal invasion range from shaving the trachea to various forms of tracheal resection. Tracheal shaving is commonly performed for tumors with minimal invasion of the perichondrium, and it consists of sharp removal of all gross disease from the surface of the trachea while leaving the mucosa intact.[4, 5] Shave excision harbors a high risk of microscopic residual disease,[6-8] A number of studies reported similar survival and local control rates for shave resections and more aggressive procedures in carefully selected patients.[9-11] That finding, however, has been called into question by the demonstration of a worse survival and a higher recurrence rate in shave resections, despite adjuvant radioactive iodine (RAI) and external beam radiotherapy.[12, 13]

The aim of this study was to compare outcomes of minimal tracheal invasion to other forms of ETE, and to analyze outcomes according to the eighth edition of the American Joint Committee on Cancer (AJCC) staging manual.

\section{Materials and Methods}

\section{Patients}

Patients with a final diagnosis of WDTC who were treated at the Tel-Aviv 'Sourasky' Medical Center between January $1^{\text {st }} 2006$ and December $31^{\text {st }} 2015$ were identified from the institutional electronic database. Demographic, clinical, pathologic, and radiologic data on all included patients were extracted. Inclusion criteria were all patients diagnosed with WDTC and confirmed ETE as described in the operative and pathological reports. All patients had undergone surgery that included at least a total thyroidectomy as a first procedure, or a subsequent completion thyroidectomy. The tracheal invasion group included only patients diagnosed with invasion stage I and II according to Shin et al.[14]. All patients in the tracheal invasion group had undergone a shave resection of the trachea. Patients with other forms of ETE (e.g., strap muscles, RLN, esophagus, larynx, and subcutaneous soft tissue) that had undergone a thyroidectomy with excision of all gross extra-thyroidal disease were included in the non-tracheal invasion group. Patients were excluded from the study for the following reasons: insufficient preoperative investigational measures (that included at least a neck ultrasound and a fine-needle aspiration), pathologies other than WDTC , undergoing surgical procedures that did not include at least a total t or completion thyroidectomy, partial resections of gross disease with residual macroscopic disease (R2 resection), minimal ETE (e.g. focal ETE detected only on histological examination), ETE invading only peri-thyroidal fat [15], or tracheal resection procedures (window or full circumferential resection).

\section{Definitions}

Tracheal invasion stage I (Shin I) was defined as disease invading through the capsule of the thyroid gland and abutting but not invading the external perichondrium of the trachea.[14] Tracheal invasion stage II (Shin II) was defined as invasion of the disease between the rings of the cartilage or as invasion such as to cause minor cartilage destruction.[14] Minimal ETE was defined as peri-thyroidal fat involvement diagnosed on final pathology without strap muscle or neck structure invasion according to the latest revision $\left(8^{\text {th }}\right.$ edition) of the AJCC staging manual.[15] Gross ETE was defined according to the updated AJCC as including any strap muscle, subcutaneous soft tissue, larynx, trachea, esophagus, and RLN involvement.[15] Margins were considered R0 if frozen section analysis and/or final pathology confirmed that the soft tissue or cartilaginous margins were tumor-free.[16] Margins were considered microscopically invaded (R1) if histological analysis showed tumor at the margin of the operating specimen or $<1 \mathrm{~mm}$ from the margin.[16] Dosimetry parameters of the delivered RAI were retrieved from treatment plans. 
Local control was defined according to the 2015 American Thyroid Association Management Guidelines for Adult Patients with Thyroid Nodules and Differentiated Thyroid Cancer:[17] An "excellent" response was defined as no clinical, biochemical, or structural evidence of disease. A "biochemical incomplete" response was defined as abnormal thyroglobulin or rising anti-thyroglobulin antibody levels in the absence of localizable disease. A "structural incomplete" response was defined as persistent or newly identified loco-regional or distant metastases. Event-free survival was defined as survival without macroscopic local, regional, or distant disease visible on imaging, with or without persistent stable biochemical disease. Disease-free survival (DFS) was defined as survival without any evidence of disease at any site. OS was defined as death due to any cause. The treatment termination date was used as time point zero.

\section{Statistical analyses}

Structural and biochemical recurrences were estimated using the competing risk methods. DFS and OS were estimated using the Kaplan-Meier method. The significance of predictors, such as biochemical control, structural control, and OS, were assessed in univariate and multivariate analyses using cox proportional hazard models. Statistical analyses using cox regression models were also performed to compare the rates of biochemical recurrence, structural recurrence, and OS between patients with tracheal involvement versus other forms of ETE. Dosimetry parameters were compared using unpaired t-tests. A two-sided $p$-value $<0.05$ was considered statistically significant for all measures

\section{Methods}

Subgroup analysis was performed on three distinct groups (staging in brackets is according to the AJCC $8^{\text {th }}$ edition on thyroid cancer):

1. Gross ETE involving only strap muscles (T3b)

2. Gross ETE with minimal tracheal invasion with or without strap muscle involvement (T4a)

3. Gross ETE with involvement of neck structures (RLN, larynx, esophagus, subcutaneous tissue) with or without tracheal invasion (T4a)

\section{Results}

Of the 1019 consecutive patients who underwent thyroidectomy between January $1^{\text {st }} 2006$ and December $31^{\text {st }} 2015$ among whom $589(95.4 \%)$ were diagnosed with WDTC. A total of 132 (22.4\% of patients diagnosed with WDTC), met the inclusion criteria. The study group was comprised of 88 females (66.7\%) and 44 males $(33.3 \%)$, with a mean age of $53.4 \pm 16.6$ years (median 54.5 years, range 12-88; IQR [41-65]), and a mean follow-up of $39.9 \pm 31.5$ months (median 29.7 months, range 0-117.7 months; IQR [14.5-62.4]).

Twenty-seven patients (20.4\%) had strap muscle invasion. This group was comprised of 21 females $(77.7 \%)$ and 6 males $(22.3 \%)$ with a mean age of $53.5 \pm 16.7$ years (median 55 , range 12 -85 years; IQR [42-62]). The mean follow-up time was $27.1 \pm 24.9$ months (median 23.7 months, range 0-98.3 months; IQR [6.9-37.9]). The final pathological results revealed that 18 patients $(66.7 \%)$ had an $\mathrm{R} 0$ resection, and that the remaining 9 patients $(33.3 \%)$ had positive microscopic margins (R1). The baseline clinical and treatment details of the study cohort are listed in Table1.

Twenty-two patients (16.7\%) had minimal tracheal invasion. This group was comprised of 16 females $(72.7 \%)$ and 6 males $(27.3 \%$ ) with a mean age of $54 \pm 17.9$ years (median 52 , range $24-88$ years; IQR [43-65.75]). The mean follow-up time was $54.4 \pm 35.8$ months (median 48.7 months, range 0-117.7 months; IQR [22.0-86.7]). The final pathological results revealed that 16 patients $(72.7 \%)$ had an $\mathrm{R} 0$ resection, and that the remaining 6 patients $(27.3 \%)$ had positive microscopic margins (R1). The baseline clinical and treatment details of the study cohort are listed in Table1.

Eighty-three patients $(62.9 \%)$ had other forms of ETE (excluding minimal tracheal invasion). The other ETE group was comprised of 50 females $(60.2 \%)$ and 33 males $(39.8 \%)$ with a mean age of $53.3 \pm 16.4$ years (median 56 years; range: 17-83, IQR [41-66]). The mean follow-up time was $40.2 \pm 30.9$ months (median 29.6 months; range 0.1-109.9 IQR [13.8-63.6]). The final pathological results revealed that 60 patients $(72.3 \%)$ 
had an $\mathrm{R} 0$ resection, and that the remaining 23 patients $(27.7 \%)$ had positive microscopic margins (R1) (Table 1).

The statistical analysis did not reveal any significant differences between the minimal tracheal ETE group, strap muscle, and the non-tracheal ETE group in patient's age, extent of surgery, or length of follow-up (Table 1). The final pathological analysis did not reveal any significant difference $(p=0.84)$ in R1 results between the non-tracheal ETE group $(n=23 ; 27.7 \%)$ and the minimal tracheal ETE group $(n=6 ; 27.3 \%)$ and strap muscle group $(\mathrm{n}=9,33.3 \%)$. All other pathological parameters, including lymph node involvement, multifocal disease, and perivascular and perineural invasion were not significant different between the tracheal and non-tracheal ETE groups (Table 1).

Postoperative RAI treatment was delivered to $15 / 22$ patients $(68.2 \%)$ in the minimal tracheal ETE group (average dose $118.81 \pm 107.60 \mathrm{mCi}$ ) , $15 / 27(55.6 \%$, avarage dose of $78.13 \pm 73.85$ ) in the t3b (strap muscle involvement, and 55/81 patients $(67.9 \%)$ in the non-tracheal t4a ETE group (average dose $140.77 \pm 154.36$ $\mathrm{mCi})(p=\mathrm{NS})$ (Table 1). Six patients (6/81) in the \souttracheal gr non-tracheal t4a ETE group underwent radiation therapy ( $p=\mathrm{NS}$ ) (Table 1$)$, no patients underwent EBRT in the two other groups. Recurrence was diagnosed in two patients $(2 / 27)$ in the strap muscle (t3b) group; 16/78 $(20.5 \%)$ in the T4a other tracheal invation group, and one patient $(1 / 22)(4.5 \%)$ in the minimal tracheal invasion group ( $p=\mathrm{NS})$. (Table 1$)$

Kaplan-Meier models that analyzed the difference in DFS (structural recurrence) between T3b ETE disease (strap muscle involvement) to T4a shave and T4a other ETE (other structural disease) did not yield any significant differences for the overall population (all ages) between the tracheal invasion group and the other forms of ETE ( $p=0.084$; Fig. 1A, Table 1).

One subgroup analysis of patients $>55$ years of age that compared structural recurrence (DFS) in T3b ETE disease (strap muscle involvement) to T4a shave and other T4a (RLN, larynx, esophagus, subcutaneous tissue) also did not reach a level of significance, but there was a strong trend towards significance ( $p=0.064$; Fig. 1B, Table 2).

Another subgroup analysis that involved combining T3b patients with T4a shave patients and comparing them to other T4a ETE patients (RLN, larynx, esophagus, subcutaneous tissue) revealed that the former performed significantly better than the latter $[p=0.028$ for all ages; $p=0.021$; for patients $>55$ years of age (Fig.1 C and D, respectively; Tables 3 and 4] in terms of structural recurrence (DFS).

\section{Discussion}

The aim of this study was to compare outcomes of minimal tracheal invasion to the other forms of ETE, namely, strap muscle, RLN, esophagus and larynx invasion. Our results demonstrated that minimal tracheal invasion undergoing shave resection, has similar outcomes to strap muscle invasion as opposed to the other forms of ETE. The analyses of the various outcomes according to the updated AJCC, revealed that minimal tracheal invasion may be more accurately staged as T3b rather than T4a.

The conflicting results of studies on shave resection outcomes might be attributed to inconsistencies of the study designs, such as varying definitions of invasion, completeness of resection, various histology and adjuvant treatments, and, most importantly, the lack of clear patient selection criteria for shave excision.[18] Shave excision for extensive tumors is likely to result in incomplete resection and should be rejected outright. In cases of minimal tracheal invasion (defined as Shin I and II), however, shave resection is a reasonable option and one that apparently has good outcomes. We found a $14 \%$ biochemical and structural recurrence rate with shave resection of the trachea. The OS in the shave group with a median follow-up of $54.4 \pm 35.8$ months was $100 \%$. These results are comparable to those in papers that showed recurrence rates ranging from 5-17\%.[19, 20] In fact, the latest (2014) American Head and Neck Society (AHNS) consensus statement stipulates that a tracheal shave excision is appropriate if a short segment of the trachea is invaded and there is minimal cartilage invasion (section 6A).[4] Judicious management of the patients, including appropriate postoperative RAI and intense follow-up, is essential to achieve such excellent results.

One of the endpoints of this study was to analyze the new AJCC staging system for thyroid cancer. We 
aimed to determine if all forms of tracheal invasion should be automatically categorized as T4a, or if the degree of visceral invasion should influence stage definition. With the caveat of not having reached a level of statistical significance, most probably as the result of the under-power of this study, the trend of minimal tracheal invasion to "behave" more like a T3b disease warrants further investigation. This trend (Fig. 1) yielded surprising results by demonstrating that patients with minimal tracheal invasion whom are considered T4a, have as good as, if not better, prognosis compared to T3b patients. Since extrapolation of this trend seemed promising, we performed another subgroup analysis in which we combined the patients with T3b and T4a shave. The results were significant and persuasive; they showed that patients with minimal tracheal invasion and/or strap muscle involvement have a better prognosis compared to patients with other structural involvement in the neck (Fig. 1). These results were robust for the all ages groups as well as for the 55-year and over age group.

In the international multi-institutional validation of age 55 years as a cutoff for risk stratification in the AJCC/UICC staging system for WDTC,[21] the authors concluded that 55 years (compared to 45 years in the older version) improved the prognostic accuracy of the TNM staging model. This conclusion was valid for the various $\mathrm{T}$ and $\mathrm{N}$ stages, and it improved the distribution of outcomes between stages I and IV disease and led to a down-staging of a significant percentage (12\%) of patients. If, indeed, such be the case, and minimal tracheal invasion has the same prognosis as strap muscle involvement, patients older than 55 years might be more correctly staged as stage 2 rather than stage 3 in the updated AJCC.

The precise biological basis for these results are not within the scope of this study, but we can speculate that very low volume residual disease in the minimal tracheal invasion group combined with proper postoperative treatment plays a major role. The fact that the other ETEs had higher rates of R1 resections compared to the shave group ( $p=0.022)$ also serves to explain their worse outcomes. The better outcomes of the shave group correspond with earlier reports that patients with ETE to the trachea with R1 resections have essentially the same prognosis as after $\mathrm{R} 0$ resections.[22]

The other end result of the current study is the relatively good DFS of the entire cohort, despite achieving a "non-oncological" surgical resection. Adjuvant RAI has been shown to decrease recurrence rates in WDTC.[23-25] Tuttle et al .[26] reported that even when post-RAI whole-body scans showed residual disease in the neck, RAI treatment resulted in a 70\% loco-regional control rate. Furthermore, the findings of several reports suggested that RAI treatment after primary surgery may also improve OS in patients with high-risk features, such as ETE.[27, 28]

There are obvious limitations to this study that bear mention. The most important shortcoming is the fact that we did not reach a level of statistical significance to prove that patients with minimal tracheal invasion have a more favorable outcome compared to other T4a patients among various age groups. The retrospective nature of this study and the relatively short follow-up time for WDTC are additional drawbacks. We were, however, able to show that patients with minimal tracheal invasion combined with strap muscle involvement had a significantly better outcome compared to other forms of ETE. We believe that these results warrant further study to elucidate whether this trend will prove valid in an appropriately powered investigation and that they may serve to downstage thousands of patients worldwide.

In summary, patients with minimal tracheal invasion who undergo shave resection had outcomes similar to patients with strap muscle invasion. They may have a better prognosis than patients with other forms of ETE. If that proves to be correct, patients with minimal tracheal invasion should be staged as T3b rather than T4a (AJCC staging system).

\section{REFRENCES}

1. Shah, J.P., et al., Prognostic factors in differentiated carcinoma of the thyroid gland. The American journal of surgery, 1992.164 (6): p. 658-661.

2. Andersen, P.E., et al., Differentiated carcinoma of the thyroid with extrathyroidal extension. The American journal of surgery, 1995.170 (5): p. 467-470. 
3. Hay, I.D., W.M. McConahey, and J.R. Goellner, Managing patients with papillary thyroid carcinoma: insights gained from the Mayo Clinic's experience of treating 2,512 consecutive patients during 1940 through 2000. Transactions of the American Clinical and Climatological Association, 2002. 113 : p. 241.

4. Shindo, M.L., et al., Management of invasive well-differentiated thyroid cancer: An American head and neck society consensus statement: AHNS consensus statement. Head \& neck, 2014.36 (10): p. 1379-1390.

5. Honings, J., et al., The management of thyroid carcinoma invading the larynx or trachea. The Laryngoscope, 2010.120 (4): p. 682-689.

6. Frazell, E.L. and F.W. Foote Jr, Papillary cancer of the thyroid. A review of 25 years of experience. Cancer, 1958.11 (5): p. 895-922.

7. Melliere, D.J., et al., Thyroid carcinoma with tracheal or esophageal involvement: limited or maximal surgery? Surgery, 1993.113 (2): p. 166-172.

8. Keum, K.C., et al., The role of postoperative external-beam radiotherapy in the management of patients with papillary thyroid cancer invading the trachea. International Journal of Radiation Oncology* Biology* Physics, 2006. 65 (2): p. 474-480.

9. Cody, H.S. and J.P. Shah, Locally invasive, well-differentiated thyroid cancer: 22 years' experience at Memorial Sloan-Kettering Cancer Center. The American Journal of Surgery, 1981. 142 (4): p. 480-483.

10. Czaja, J.M. and T.V. McCaffrey, The surgical management of laryngotracheal invasion by well-differentiated papillary thyroid carcinoma. Archives of Otolaryngology-Head \& Neck Surgery, 1997.123 (5): p. 484-490.

11. Nishida, T., K. Nakao, and M. Hamaji, Differentiated thyroid carcinoma with airway invasion: indication for tracheal resection based on the extent of cancer invasion. The Journal of thoracic and cardiovascular surgery, 1997. 114 (1): p. 84-92.

12. Park, C.S., K.W. Suh, and J.S. Min, Cartilage-shaving procedure for the control of tracheal cartilage invasion by thyroid carcinoma. Head \& neck, 1993. 15 (4): p. 289-291.

13. Kasperbauer, J.L., Locally advanced thyroid carcinoma. Annals of Otology, Rhinology \& Laryngology, 2004. 113 (9): p. 749-753.

14. Shin, D.-H., et al., Pathologic staging of papillary carcinoma of the thyroid with airway invasion based on the anatomic manner of extension to the trachea: a clinicopathologic study based on 22 patients who underwent thyroidectomy and airway resection. Human pathology, 1993. 24 (8): p. 866-870.

15. Tuttle, R.M., B. Haugen, and N.D. Perrier, Updated American Joint Committee on Cancer/Tumor-NodeMetastasis Staging System for differentiated and anaplastic thyroid cancer: what changed and why?2017, Mary Ann Liebert, Inc. 140 Huguenot Street, 3rd Floor New Rochelle, NY 10801 USA.

16. Hartl, D.M., et al., Resection margins and prognosis in locally invasive thyroid cancer. Head \& neck, 2014. 36 (7): p. 1034-1038.

17. HaugenBryan, R., et al., 2015 American Thyroid Association management guidelines for adult patients with thyroid nodules and differentiated thyroid cancer: the American Thyroid Association guidelines task force on thyroid nodules and differentiated thyroid cancer. Thyroid, 2016.

18. Chernichenko, N. and A.R. Shaha, Role of tracheal resection in thyroid cancer. Current opinion in oncology, 2012. 24 (1): p. 29-34.

19. McCarty, T.M., et al., Surgical management of thyroid cancer invading the airway. Annals of surgical oncology, 1997. 4 (5): p. 403-408.

20. Tsukahara, K., I. Sugitani, and K. Kawabata, Surgical management of tracheal shaving for papillary thyroid carcinoma with tracheal invasion. Acta oto-laryngologica, 2009. 129 (12): p. 1498-1502. 
21. Nixon, I.J., et al., An international multi-institutional validation of age 55 years as a cutoff for risk stratification in the AJCC/UICC staging system for well-differentiated thyroid cancer.Thyroid, 2016. 26 (3): p. $373-380$.

22. Brauckhoff, M., et al., Impact of extent of resection for thyroid cancer invading the aerodigestive tract on surgical morbidity, local recurrence, and cancer-specific survival. Surgery, 2010.148 (6): p. 1257-1266.

23. Mazzaferri, E.L. and S.M. Jhiang, Long-term impact of initial surgical and medical therapy on papillary and follicular thyroid cancer. The American journal of medicine, 1994. 97 (5): p. 418-428.

24. Sawka, A.M., et al., An updated systematic review and commentary examining the effectiveness of radioactive iodine remnant ablation in well-differentiated thyroid cancer. Endocrinology and metabolism clinics of North America, 2008. 37 (2): p. 457-480.

25. Tuttle, R.M., G. Rondeau, and N.Y. Lee, A risk-adapted approach to the use of radioactive iodine and external beam radiation in the treatment of well-differentiated thyroid cancer. Cancer Control, 2011.18 (2): p. $89-95$.

26. Tuttle, R.M., et al., Radioactive iodine administered for thyroid remnant ablation following recombinant human thyroid stimulating hormone preparation also has an important adjuvant therapy function.Thyroid, 2010. 20 (3): p. 257-263.

27. Samaan, N.A., et al., The results of various modalities of treatment of well differentiated thyroid carcinomas: a retrospective review of 1599 patients. The Journal of Clinical Endocrinology \& Metabolism, 1992. 75 (3): p. 714-720.

28. Jonklaas, J., et al., Outcomes of patients with differentiated thyroid carcinoma following initial therapy. Thyroid, 2006.16 (12): p. 1229-1242.

\section{FIGURE LEGEND}

Figure 1: Kaplan Meier (KM) curves;

(A) KM for recurrence, all ages, 3-group analysis [blue = T3b (strap muscle involvement); green = T4a (minimal tracheal involvement); red = T4a (RLN, larynx, esophagus or subcutaneous tissue involvement)]

(B) KM for recurrence, age $>55$, 3-group analysis [blue $=$ T3b (strap muscle involvement); green $=$ T4a (minimal tracheal involvement); red = T4a (RLN, larynx, esophagus or subcutaneous tissue involvement)]

(C) KM for recurrence, all ages, 2-group analysis [blue = T3b (strap muscle involvement) + T4a (minimal tracheal involvement); red = T4a (RLN, larynx, esophagus or subcutaneous tissue involvement)]

(D) KM for recurrence, age $>55$, 2-group analysis [blue $=$ T3b (strap muscle involvement) + T4a (minimal tracheal involvement); red = T4a (RLN, larynx, esophagus or subcutaneous tissue involvement)] 

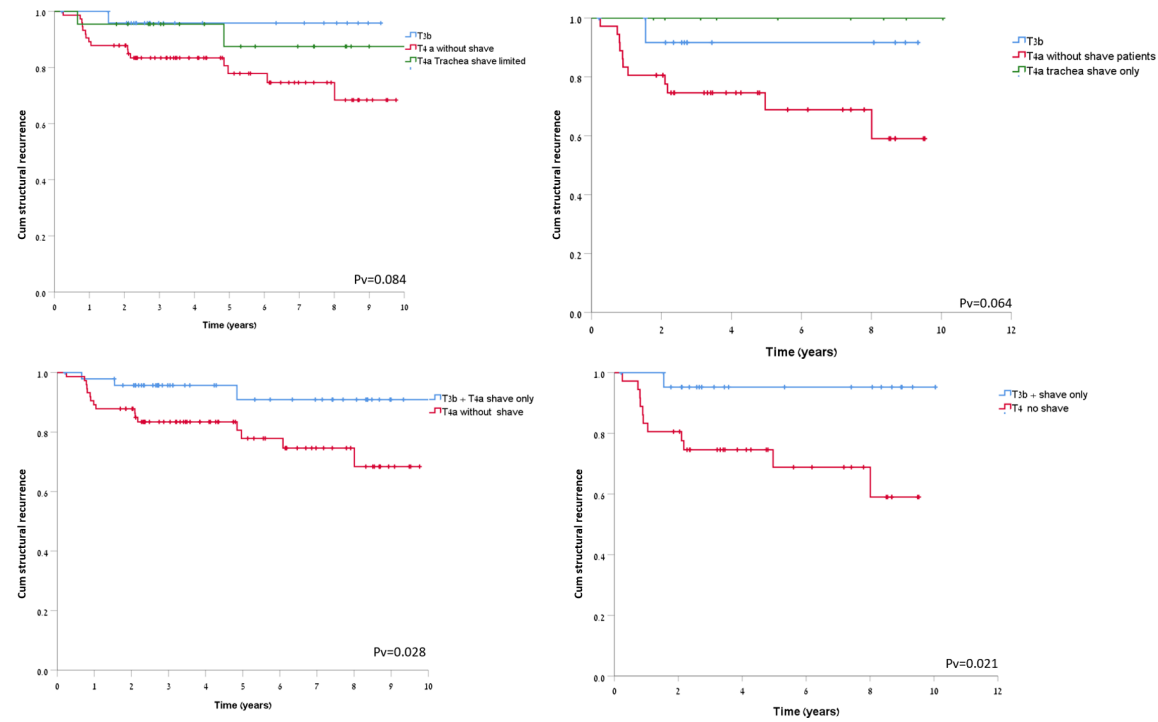

\section{Hosted file}

tables (5). docx available at https://authorea.com/users/309373/articles/440314-better-than-expectedprognosis-of-minimal-tracheal-invasion-in-well-differentiated-thyroid-carcinoma 